\title{
Exonic WT1 pathogenic variants in 46,XY DSD associated with gonadoblastoma
}

\author{
Sneha Arya ${ }^{1, *}$, Sandeep Kumar ${ }^{1, *}$, Anurag R Lila@1, Vijaya Sarathi ${ }^{2}$, Saba Samad Memon ${ }^{1}$, Rohit Barnabas ${ }^{1}$, \\ Hemangini Thakkar ${ }^{3}$, Virendra A Patil ${ }^{1}$, Nalini S Shah ${ }^{1}$ and Tushar R Bandgar ${ }^{1}$ \\ 'Department of Endocrinology, Seth G S Medical College \& KEM Hospital, Mumbai, India \\ ${ }^{2}$ Department of Endocrinology, Vydehi Institute of Medical Sciences and Research Centre, Bangalore, India \\ ${ }^{3}$ Department of Radiology, Seth G S Medical College \& KEM Hospital, Mumbai, India
}

Correspondence should be addressed to A R Lila: anuraglila@gmail.com

*(S Arya and S Kumar contributed equally to this work)

\begin{abstract}
Objective: The literature regarding gonadoblastoma risk in exonic Wilms' tumor suppressor gene (WT1) pathogenic variants is sparse. The aim of this study is to describe the phenotypic and genotypic characteristics of Asian-Indian patients with WT1 pathogenic variants and systematically review the literature on association of exonic WT1 pathogenic variants and gonadoblastoma.

Design: Combined retrospective-prospective analysis.

Methods: In this study, 46,XY DSD patients with WT1 pathogenic variants detected

by clinical exome sequencing from a cohort of 150 index patients and their affected relatives were included. The PubMed database was searched for the literature on gonadoblastoma with exonic WT1 pathogenic variants.

Results: The prevalence of WT1 pathogenic variants among 46,XY DSD index patients was $2.7 \%$ (4/150). All the four patients had atypical genitalia and cryptorchidism. None of them had Wilms' tumor till the last follow-up, whereas one patient had late-onset nephropathy. 11 p13 deletion was present in one patient with aniridia. The family with p.Arg458GIn pathogenic variant had varied phenotypic spectrum of Frasier syndrome; two siblings had gonadoblastoma, one of them had growing teratoma syndrome (first to report with WT1). On literature review, of $>100$ exonic point pathogenic variants, only eight variants (p.Arg462Trp, p.Tyr177*, p.Arg434His, p.Met410Arg, p.GIn142*, p.Glu437Lys, p.Arg458*, and p.Arg458GIn) in WT1 were associated with gonadoblastoma in a total of 15 cases (including our two cases).

Conclusions: WT1 alterations account for $3 \%$ of $46, X Y$ DSD patients in our cohort. $46, X Y$ DSD patients harboring exonic WT1 pathogenic variants carry a small but definitive risk of gonadoblastoma; hence, these patients require a gonadoblastoma surveillance with a more stringent surveillance in those harboring a gonadoblastoma-associated variant.
\end{abstract}

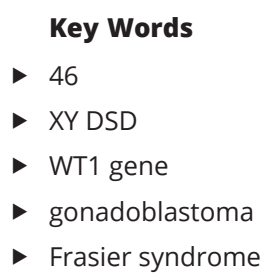

Endocrine Connections (2021) 10, 1522-1530

\section{Introduction}

Wilms' tumor suppressor gene (WT1), located on chromosome $11 \mathrm{p} 13$, encodes a zinc-finger protein. It contains ten exons and spans about $50 \mathrm{~kb}$ (1). Exons 1-6 encode a proline/glutamine-rich transcriptionalregulation region, and exons $7-10$ encode the four zinc fingers of the DNA-binding domain (2). Pathogenic alterations of this transcription factor cause various disorders of urogenital development. There is a broad phenotypic spectrum with WT1 pathogenic variants. Microdeletions (including the PAX6 gene) lead to 
WAGR syndrome (OMIM 194072), a complex phenotype associated with Wilms' tumor, aniridia, genitourinary anomalies and mental retardation (3). Exonic variants are responsible for Denys-Drash syndrome (DDS, OMIM 194080), comprising XY gonadal dysgenesis, predisposition to nephropathy, and Wilms' tumors (4). Five percent of patients with sporadic Wilms' tumors have WT1 pathogenic variants, whereas $>90 \%$ of DDS patients harbor exonic WT1 pathogenic variants (5). Donor splice-site mutations (downshifting of the ratio of +KTS (lysine, threonine, and serine) to -KTS isoforms) of intron 9 are responsible for Frasier syndrome (FS, OMIM 136680), comprising of $\mathrm{XY}$ gonadal dysgenesis and predisposition to gonadoblastoma and nephropathy (6). Wilm's tumor is not typically seen in patients with FS. Very few exonic pathogenic variants have been described with FS to date (7). While the risk of Wilm's tumor is well described, the literature regarding gonadoblastoma risk in exonic $W T 1$ pathogenic variants is sparse (8). In this study, we describe the phenotypic and genotypic spectrum of WT1 pathogenic variants from a large cohort of Asian-Indian 46,XY DSD. Additionally, a systematic literature review was done to describe characteristics of patients having gonadoblastoma associated with exonic WT1 pathogenic variants.

\section{Materials and methods}

This study is a part of the research project titled 'genotype-phenotype correlation in Indian patients with 46,XY DSD' approved by the Institutional Ethics Committee II of Seth GS Medical College (EC/ OA-47/2014), Mumbai. Consecutive index patients with 46 , XY DSD having atypical or female external genitalia registered between January 2005 and August 2020 were included in the study. Patients presenting with isolated hypospadias (distal and mid) or isolated cryptorchidism (unilateral/bilateral) with typically male genitalia were excluded. Genetic analysis was done after obtaining the written informed consent. Wherever available, family members of the index cases with pathogenic variations were offered genetic analysis for the observed pathogenic variant.

Demographic characteristics (age at presentation and evaluation, family history of DSD, and sex assignment), clinical findings (external genitalia development grading by Sinnecker score), hormonal profile, ultrasonogram of abdomen and pelvis, and histopathology of gonads were recorded (9).
Genomic DNA was isolated from peripheral blood leukocytes by standard techniques. Molecular screening of the WT1 gene was performed by next-generation sequencing. Targeted gene capture was done using a custom capture kit. The libraries were sequenced to mean $>80-100 X$ coverage on the Illumina sequencing platform. The coverage for the WT1 gene was 100X. We followed the GATK best practices framework for the identification of variants in the sample using Sentieon (v201808.01). All the variants were confirmed by Sanger sequencing. The observed variants were classified as per American College of Medical Genetics and Genomics guidelines. Sequence variants were designated using the reference sequences of WT1 isoform D, NM_024426.4 (cDNA), and NP_077744.3 (protein). Splicing effects of exonic variants associated with gonadoblastoma were tested using in silico splicing prediction tools, Netgene2 and NNsplice, and were compared with wild type.

Serum follicle-stimulating hormone (FSH), luteinizing hormone (LH), and total testosterone (T) levels were measured by chemiluminescence immunoassay (ADVIA Centaur XP Immunoassay Systems, Siemens). Human chorionic gonadotropin (hCG)-stimulated $\mathrm{T}$ values were evaluated after three daily doses (1500 IU) of i.m. hCG. Anti-Mullerian hormone (AMH) levels were measured by ELISA. Intra-assay and inter-assay coefficients of variation were less than $10 \%$ for all the hormonal assays.

\section{Systematic review of literature}

The PubMed database (English language) was searched in September 2021 using the keywords 'WT1 mutation AND gonadoblastoma' OR 'Denys-Drash syndrome' OR 'Frasier syndrome'. A total of 413 publications were screened. Cross-references of selected publications and review articles were used to find additional articles. After exclusion for various reasons (as detailed in Fig. 1), 12 articles (13 patients) had histologically proven gonadoblastoma associated with exonic pathogenic variants in WT1 and were thus included. Patients' data were tabulated to include details of phenotype, genotype, and characteristics of associated tumors and/or nephropathy.

\section{Statistical analysis}

The categorical data were expressed as actual numbers and percentages. Quantitative data were expressed as range.

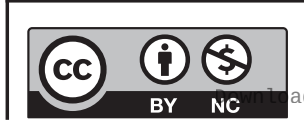

This work is licensed under a Creative Commons Attribution-NonCommercial 4.0 International License. ded from Bioscientifica.com at 04/26/2023 12:12:56AM 

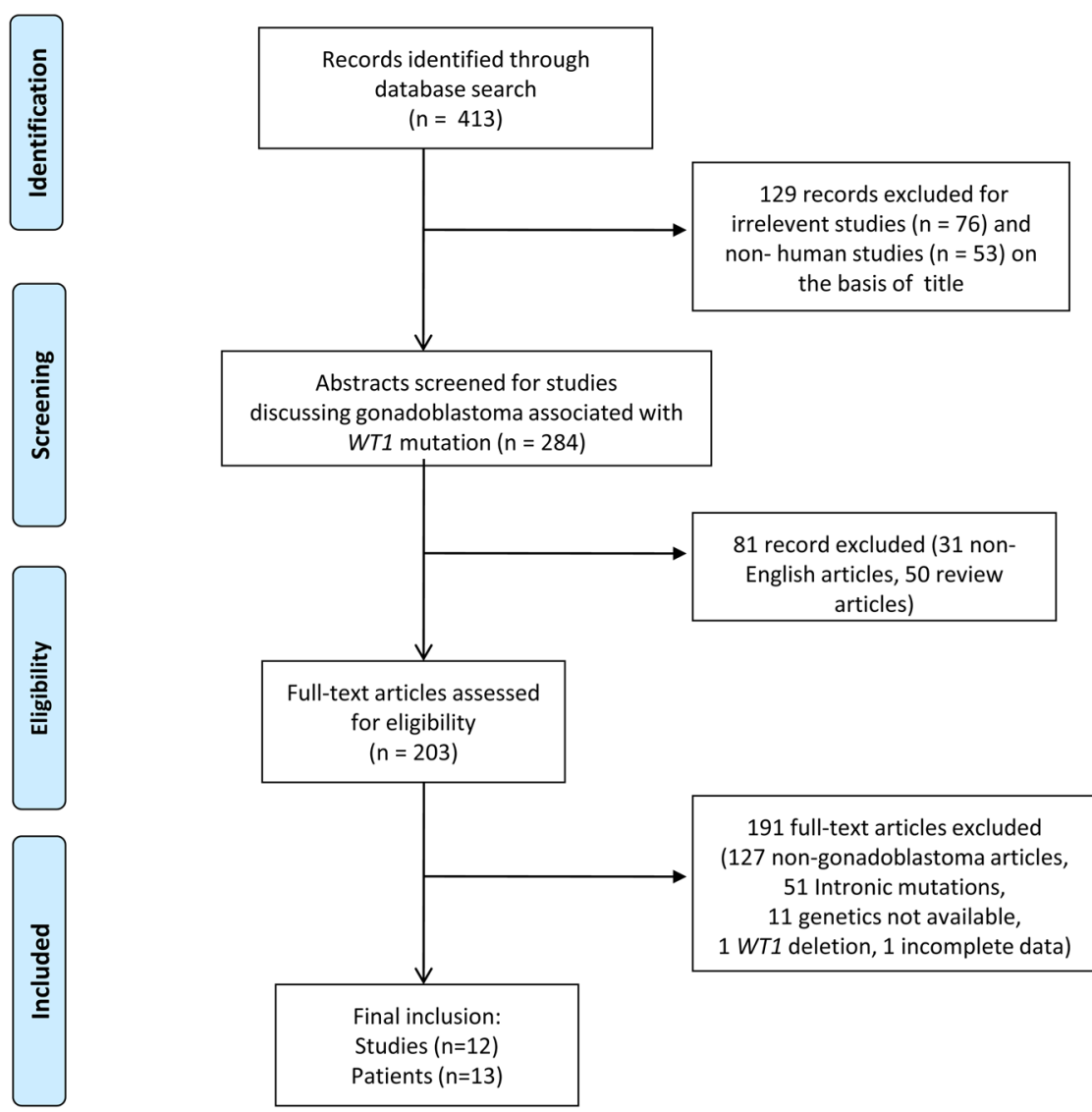

Figure 1

PRISMA flow chart for literature search of gonadoblastoma associated with WT1 exonic pathogenic variants.

\section{Results}

Four (2.7\%) of the 150 index patients of $46, \mathrm{XY}$ DSD had pathogenic variants in WT1. Cryptorchidism (unilateral/ bilateral) was noted in 82 of the 150 index patients, and all four $(4 / 82,4.9 \%)$ patients with WT1 variants belonged to this group. An affected family member (P4b) with 46,XY DSD was also included for the phenotypic analysis.

The clinical findings, hormonal profile, and genetic data of patients from our cohort with pathogenic variants in $W T 1$ are summarized in Table 1 . Three patients (P1-P3) presented at prepubertal age (0.5-7 years) with atypical genitalia (Sinnecker score 2-3) and cryptorchidism were reared as males. P1 had an additional finding of aniridia. None of them had proteinuria or Wilms' tumor till the last follow-up. Ultrasonogram did not show gonadoblastoma, but gonadal histology was not available for these patients. Diagnostic laparoscopy for the non-localized gonads, orchidopexy with biopsy of the localized gonads, and male genitoplasty, as appropriate, have been planned in these three patients. The patient with aniridia (P1) had contiguous $11 \mathrm{p} 13$ deletion comprising of WT1 and PAX6 genes. P2 had a de novo pathogenic variant,
p.Arg462Trp. P3 was found to have a maternally inherited pathogenic variant, p.Arg458Gln.

P4a was a familial case with an affected sibling. He presented at 26 years with complaints of gynecomastia and atypical genitalia (Sinnecker score 3). At initial evaluation, he was hypertensive $(170 / 100 \mathrm{mmHg})$ and had microalbuminuria (urine dipstick for albumin: trace). On follow-up at 28 years of age, his spot urine albumin/ creatinine ratio: $2360 \mu \mathrm{g} / \mathrm{mg}$ (normal $<30 \mu \mathrm{g} / \mathrm{mg}$ ) was suggestive of macroalbuminuria. His serum creatinine: $1.0 \mathrm{mg} / \mathrm{dL}$ (eGFR: $92.6 \mathrm{~mL} / \mathrm{min} / 1.73 \mathrm{~m}^{2}$ ) was normal. He underwent bilateral mastectomy and male genitoplasty with the laparoscopic bilateral gonadectomy and removal of Mullerian structures in view of non-functioning gonads and risk of malignancy. Histopathology of the left gonad was suggestive of gonadoblastoma, whereas the right gonad was dysgenetic. Patient was started on testosterone replacement therapy. This patient had an FS phenotype (46,XY gonadal dysgenesis, late-onset nephropathy, and gonadoblastoma). His younger sister (P4b) was 46,XY DSD, presented with primary amenorrhea at 17 years of age and had female external genitalia with normal breast development on examination. At presentation, her gonads were non-localizable on an ultrasonogram.

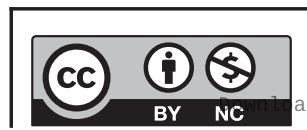




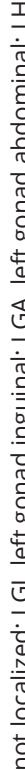

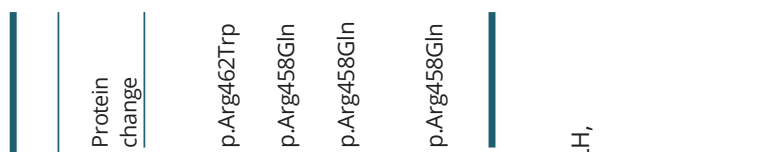

Six months later, she presented with abdominal pain and was found to have a right-sided pelvic mass $(17 \times 15 \times 16 \mathrm{~cm})$. Tumor markers including serum lactate dehydrogenase, $\alpha$-fetoprotein $(\alpha \mathrm{FP}), \mathrm{hCG}$, and cancer antigen (CA) 125 were elevated. The patient underwent exploratory laparotomy, and the right pelvic mass was excised along with left gonadectomy and bilateral salpingectomy. Histopathology of the right gonadal mass was suggestive of a malignant germ cell tumor and that of the left gonad showed a dysgerminoma. Following surgery, tumor markers normalized, and chemotherapy was started. While being on chemotherapy, she developed generalized lymphadenopathy. Biopsy from an enlarged supraclavicular lymph node showed a metastatic germ cell tumor. Based on the enlarging metastatic masses despite appropriate chemotherapy and normalization of tumor markers, a diagnosis of growing teratoma syndrome was made (Fig. 2). She remained normotensive and had a normal serum creatinine of $0.71 \mathrm{mg} / \mathrm{dL}$ (eGFR: $115 \mathrm{~mL} / \mathrm{min} / 1.73 \mathrm{~m}^{2}$ ) and no albuminuria (spot urine albumin/creatinine ratio: $29 \mu \mathrm{g} / \mathrm{mg}$ ) till last follow up at 19 years of age. Hormonal evaluation at the presentation in both the siblings ( $\mathrm{P} 4 \mathrm{a}$ and $\mathrm{P} 4 \mathrm{~b}$ ) was suggestive of primary gonadal failure. At the age of 45 , their mother was found to have isolated microalbuminuria (spot urine albumin/creatinine ratio: $64 \mu \mathrm{g} / \mathrm{mg}$ ), which was reconfirmed a month later. She was normotensive and had a normal serum creatinine of $0.8 \mathrm{mg} / \mathrm{dL}$ (eGFR: $82.4 \mathrm{~mL} / \mathrm{min} / 1.73 \mathrm{~m}^{2}$ ). Her menstrual cycles were regular, and serum gonadotropins were normal for age. None of the affected family members had Wilms' tumor until the last follow-up. These three familial patients had a missense pathogenic variant, p.Arg458Gln, in exon 9.

The details of patients with gonadoblastoma associated with $W T 1$ exonic pathogenic variants reported in the literature ( $n=13)$ and in this study (one family) are summarized in Table $2(4,7,10,11,12,13,14,15,16$, $17,18,19)$. The age of presentation ranged from birth to 26 years. Gonadoblastoma was detected earliest at 7 months of age, ranging up to 28 years. Majority of the cases $(12 / 15,80 \%)$ were found to have gonadoblastoma after prophylactic gonadectomy, while in the rest $(n=3)$, gonadoblastoma was found on biopsy, autopsy, and imaging for an abdominal lump in one case each. Out of 15 patients, 9 had atypical external genitalia, and others had female external genitalia. Only 1 patient had Wilms' tumor, whereas nephropathy was observed in 12 of the 14 cases. Nephropathy was documented before 8 years of age in all the reported cases, whereas in our patient $\mathrm{P} 4 \mathrm{a}$, it was noted as microalbuminuria and hypertension at 

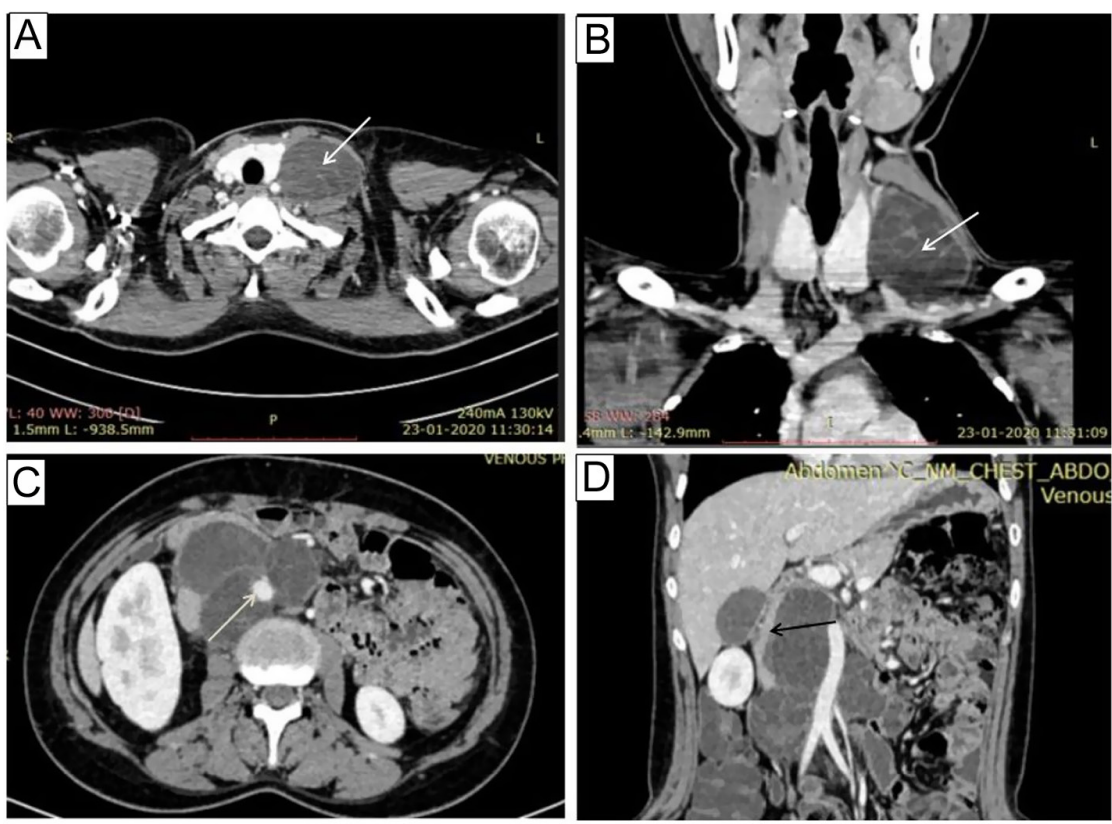

\section{Figure 2}

(A) Axial and (B) coronal contrast-enhanced CT images at the level of the neck showing a well defined enlarged left supraclavicular lymph node (white arrow) with cystic areas and few internal septae. Similar morphology lymph nodes are also seen in the para-aortic and inter-aortocaval locations in axial (C) and coronal (D) images of the abdomen. Note the lifting of the aorta (grey arrow - C) and rightward displacement of the inferior vena cava (black arrow - D) and resultant altered axis of the right kidney.

26 years. All patients had a karyotype of $46, \mathrm{XY}$ except for one patient with 47,XXY. Twelve out of the 14 index cases had pathogenic variants in DNA-binding domain (exons 7,8 , and 9). The most common exonic pathogenic variant found was p.Arg462Trp $(n=5)$ followed by p.Arg434His $(n=2)$ and p.Met410Arg $(n=2)$; others were p.Tyr177*, p.Glu437Lys, p.Arg458*, p.Arg458Gln, and p.Gln142*. On in silico splicing prediction analysis, these missense WT1 variants associated with gonadoblastoma were not found to be different from the wild type.

\section{Discussion}

This study reports $W T 1$ pathogenic variations in 4 (2.7\%) of the 150 Asian-Indian index patients with 46,XY DSD and describes an increased gonadoblastoma risk for the first time with p.Arg458Gln, a missense variant in exon 9, and a widely variable FS phenotypic spectrum in this family.

The prevalence ( 3\%) of $W T 1$ pathogenic variations in our cohort of $46, \mathrm{XY}$ DSD patients is similar to that reported (0.7-5\%) from various larger $(n \geq 40)$ cohorts from the rest of the world $(20,21,22,23)$. A typical characteristic in patients with gonadal dysgenesis is impalpable gonad(s). In our study, all four patients with WT1 variants had unilateral or bilateral cryptorchidism. Similarly, Kohler et al. reported universal occurrence of cryptorchidism in 46 ,XY DSD patients with WT1 pathogenic variants. None of the patients in our series had nongonadal manifestations at presentation except P1, who had detectable aniridia. Hence, all 46,XY DSD patients with suspected gonadal dysgenesis should undergo a formal ophthalmology examination to identify aniridia, the presence of which can guide the appropriate genetic test (11p13 deletion). Wilms' tumors were absent in all patients at presentation and during the follow-up. Nephropathy was seen in one patient, detected in the third (P4a) decade. Hence, WT1 genetic analysis should be considered in 46,XY DSD patients with gonadal dysgenesis irrespective of the other features of WT1-associated syndromes.

The phenotype in P1 (atypical genitalia and aniridia) was consistent with WAGR syndrome, the characteristic phenotype of 11p13 deletion. In a large cohort of WAGR syndrome $(n=54), 24,14$, and 11 patients had all the four, three, and two classical components, respectively. The most common feature was aniridia (98.1\%), whereas Wilms' tumor was observed in 57.4\% (3). Nevertheless, up to $45 \%$ of patients with aniridia harboring $11 \mathrm{p} 13$ deletion comprising of WT1 and PAX6 may develop Wilms' tumor (24). Hence, 11p13 deletion patients not manifesting Wilms' tumor at diagnosis should be monitored for its development. In our case, Wilms' tumor was not found till the last follow-up (2.5 years of age).

P2, who presented in infancy with atypical genitalia, had p.Arg462Trp, a hot-spot most common (39.6\%) pathogenic variant described with DDS (5). This genetic variant exhibits a broad phenotypic spectrum as reviewed by Mazen et al., where out of 15 patients, 14 (93.3\%) patients had nephropathy, 4 (26.6\%) had https://ec.bioscientifica.com https://doi.org/10.1530/EC-21-0289 (c) 2021 The authors Published by Bioscientifica Ltd

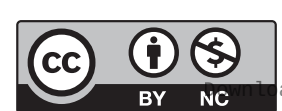

This work is licensed under a Creative Commons Attribution-NonCommercial 4.0 International License. ded from Bioscientifica.com at 04/26/2023 12:12:56AM 

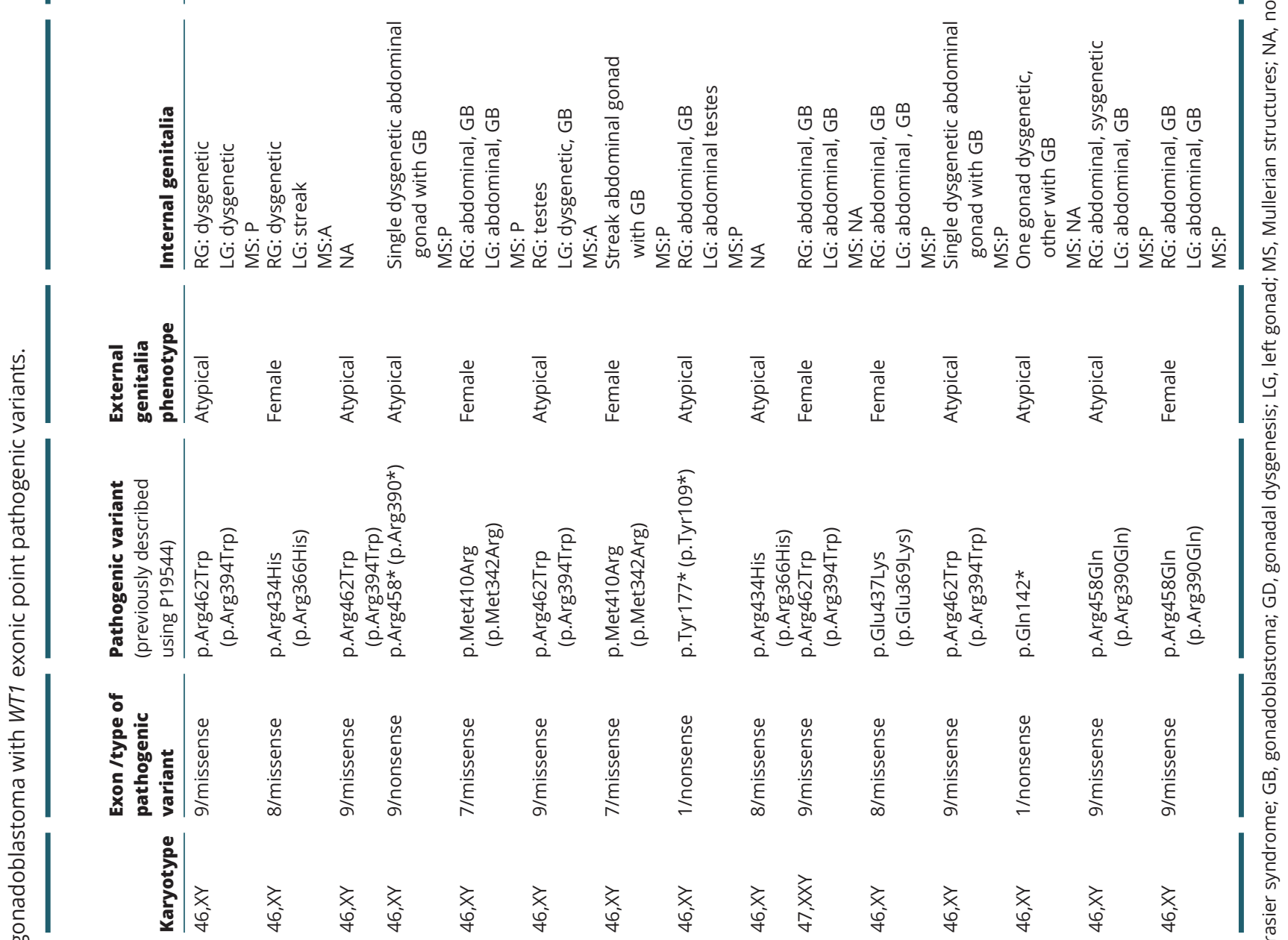

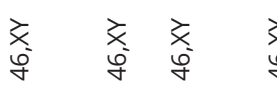

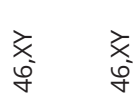

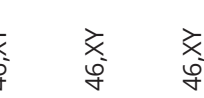

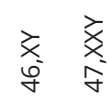

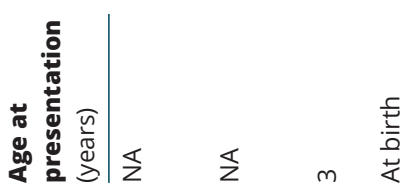

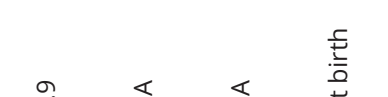

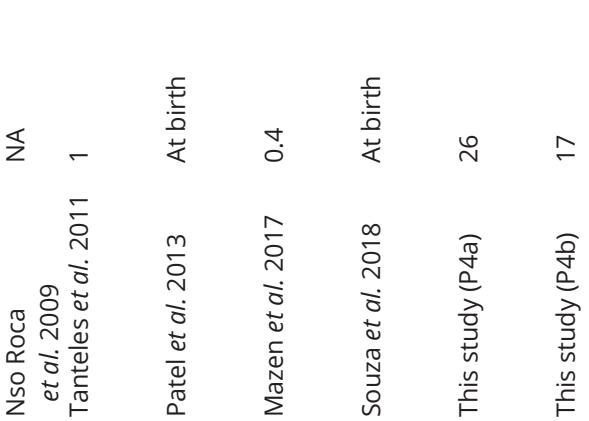
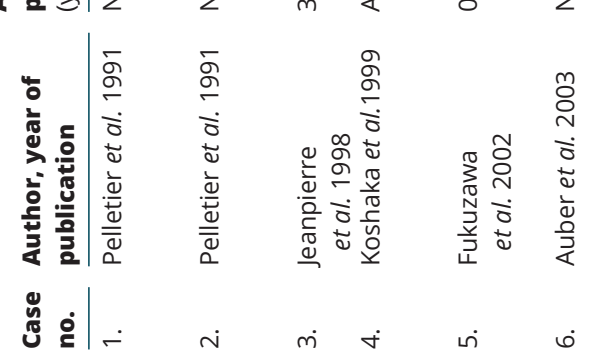

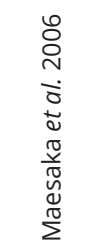

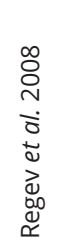

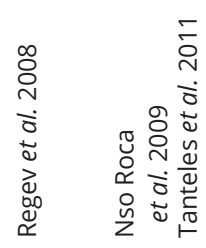

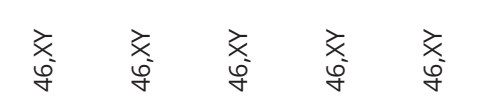

高| 
Wilms' tumor, and 2 (13.3\%) had gonadoblastoma (18). As per our literature review, p.Arg462Trp was also the most common $(5 / 14,35.7 \%)$ variant reported in patients with gonadoblastoma and exonic WT1 pathogenic variants. However, the limited follow-up did not allow this patient's (P2) complete phenotypic characterization as he did not manifest either with early nephropathy or Wilms' tumor at the last follow-up (6 months of age). p.Arg462Trp, being the most common exonic WT1 pathogenic variant associated with gonadoblastoma, P2 has been advised to undergo bilateral orchidopexy with biopsy and subsequent close surveillance for gonadoblastoma development.

A lack of definitive genotype-phenotype correlation in patients with WT1 pathogenic variants has been observed. Notably, affected XY patients with p.Arg458Gln variant ( $\mathrm{P} 3, \mathrm{P} 4 \mathrm{a}$, and $\mathrm{P} 4 \mathrm{~b}$ ) had variable phenotype. P3 and $\mathrm{P} 4 \mathrm{a}$ had partial gonadal dysgenesis, whereas $\mathrm{P} 4 \mathrm{~b}$ had complete gonadal dysgenesis. P4a had late-onset nephropathy, whereas P3 and P4b had no nephropathy till the last follow-up. P4a and $\mathrm{P} 4 \mathrm{~b}$ had gonadoblastoma, whereas gonadal histology of P3 was not available (though his ultrasonogram was normal at the last follow-up). Interestingly, P4b had spontaneous normal breast development. This may be due to the production of estrogens (serum estradiol: $370.4 \mathrm{pmol} / \mathrm{L}$ ) by streak gonads, or pre-existing gonadoblastoma, or increased sensitivity of breast tissue to estrogens (25). p.Arg458Gln has been reported previously in a Hispanic girl (karyotype not described) who developed steroid-resistant nephrotic syndrome at 10 years of age (26). Another 46,XY DSD patient with atypical genitalia (raised as female) inherited this variant from an unaffected father, showing incomplete penetrance (20). Similarly, the mother of patients $4 \mathrm{a}$ and $4 \mathrm{~b}$, who also had the same variant, had no overt manifestations but had only microalbuminuria on evaluation at 45 years of age. Notably, each of the three family members with p.Arg458Gln had different phenotypes of FS: the mother had type 3 FS (female external genitalia with 46 , XX with no gonadoblastoma risk), the elder sibling had type $2 \mathrm{FS}$ (male external genitalia with $46, \mathrm{XY}$ and gonadoblastoma risk in one-third), and the younger sibling had type 1 FS (female external genitalia with $46, \mathrm{XY}$ with gonadoblastoma risk in two-thirds) (27). These observations emphasize the wide variability in the phenotype of patients with p.Arg458Gln, even among the members of the same family.

Notably, the P4b had no localization of gonads at initial evaluation but presented with a large gonadoblastoma 6 months later. This patient also had growing teratoma syndrome after surgery and chemotherapy, suggesting an aggressive behavior, which probably explains the rapid development and progression of gonadoblastoma at diagnosis. On the other hand, this may also undermine the limitations of the commonly recommended modalities for gonadoblastoma surveillance such as ultrasonogram to identify pre-malignant lesion or small-sized malignant germ cell tumor (28). This patient is the first case of growing teratoma syndrome with WT1 pathogenic variants, to the best of our knowledge. The association of the gonadoblastoma in two of our three 46,XY DSD patients with p.Arg458Gln suggests a high risk of gonadoblastoma with this variant, suggesting a need for regular monitoring. Additionally, the rapid development and progression of gonadoblastoma in one of our patients may suggest frequent monitoring at 4-6 monthly intervals. Based on the occurrence of gonadoblastoma in P4a and P4b, P3 having the same variant was advised left-sided orchidopexy with biopsy. Further plan is to monitor the child for the development of gonadoblastoma with monthly

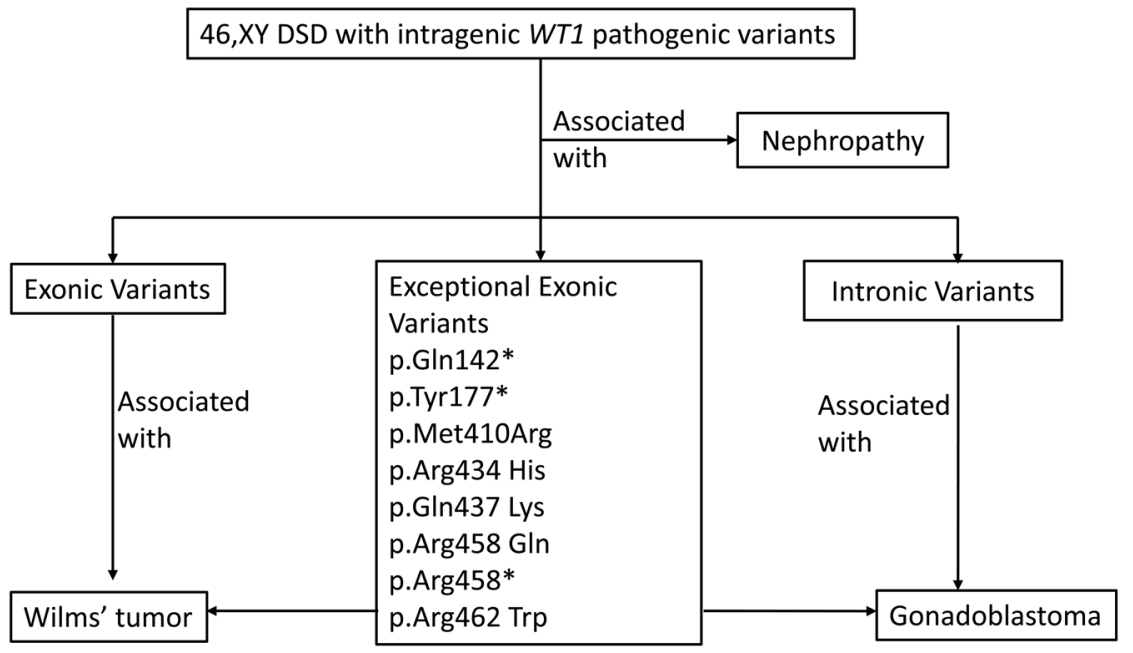

Figure 3

Schematic presentation of $46, X Y$ DSD patients with intragenic WT1 pathogenic variants and associated syndromic features. 
self-examination, and annual monitoring with ultrasonogram of gonads, and tumor markers (serum $\alpha \mathrm{FP}$ and hCG). Parents were also counseled about the limitations of these surveillance modalities (29).

In patients with intron 9 splice-site variants in WT1, those with type 1 and type 2 FS are at high risk for gonadoblastoma (27). The risk for gonadoblastoma seems to be lower in patients with nonsense or missense WT1 variants and partial gonadal dysgenesis, but current data are insufficient for guiding management (8). To date, more than 100 exonic point variants in WT1 have been reported with different phenotypes (http://www.hgmd. cf.ac.uk/ac/gene.php?gene=WT1, accessed 21/09/2021). In the literature review, we found only 13 patients having gonadoblastoma with one of the seven exonic point variants in $W T 1$, p.Arg462Trp, p.Arg458*, p.Arg434His, p.Tyr177*, p.Met410Arg, p.Gln142*, and p.Gln437Lys. Here, we report the association of p.Arg458Gln, the eighth exonic variant, with gonadoblastoma. In patients with these eight variants, we suggest stringent regular surveillance for gonadoblastoma development. We also recommend shared decision making with patient/parents for prophylactic gonadectomy after weighing the adequacy of residual gonadal function to preserve fertility and avoid the need for hormone replacement and the potential for gender dysphoria against the risk of production of opposite sex hormones and missing the early diagnosis of germ cell neoplasia due to the inherent limitations of surveillance methods (Fig. 3). Although the gonadoblastoma has been reported with only eight variants, we caution against assuming the absence of gonadoblastoma risk in 46,XY DSD patients with other exonic WT1 variants.

The intron 9 splice-site variants, the most common variants in FS patients, cause a reduction in the ratio of +KTS to -KTS WT1 isoforms (<2:1). The +KTS WT1 isoform upregulates SRY expression, which in turn upregulates SOX9 expression and thus, plays an active role in Sertoli cell differentiation and homeostasis. Decreased expression of +KTS WT1, SRY, and SOX9 affects Sertoli cell maturation and thus, their ability to support germ cell differentiation. This may result in the arrest of spermatogenesis and malignant transformation of germ cells (30). The molecular physiology of how the exonic missense variants in WT1 lead to gonadoblastoma is not clear. In human genetic disorders, a few exonic missense pathogenic variants are reported to be associated with splicing defects (31). However, on in silico analysis, no such association with splicing defects was noted for the exonic WT1 variants associated with gonadoblastoma. Hence, further cDNA and mRNA studies are warranted to evaluate the effect of
p.Arg462Trp, p.Arg434His, p.Gln437Lys, p.Met410Arg, and p.Arg458Gln on splicing defects.

The study was limited by a small number of participants, the retrospective phenotypic data collection, and a shorter duration of the follow-up. Nonetheless, this is the first report of WT1 pathogenic variants in 46,XY DSD cohort from India that reports a few novel observations in patients with WT1 pathogenic variants.

In conclusion, WT1 alterations account for 3\% of patients with $46, \mathrm{XY}$ DSD in our cohort. We report the first case of WT1-associated growing teratoma syndrome. Wide phenotypic variation may be present despite the same genotype. This study and the systematic review demonstrate a small but definitive gonadoblastoma risk in 46,XY DSD patients with exonic WT1 variants suggesting a need for gonadoblastoma surveillance in them. Of $>100$ exonic point pathogenic variants reported to date, only eight variants (p.Arg462Trp (most common), p.Tyr177*, p.Arg434His, p.Glu437Lys, p.Arg458*, p.Met410Arg, p.Gln142*, and p.Arg458Gln) in WT1 were associated with gonadoblastoma. We suggest that more stringent gonadoblastoma surveillance should be considered in $46, \mathrm{XY}$ DSD patients with these variants.

\section{Declaration of interest}

The authors declare that there is no conflict of interest could be perceived as prejudicing the impartiality of the research reported.

\section{Funding}

This work was supported by the funds from the Department of Science and Technology-Science and Engineering Research Board (DST-SERB).

\section{Acknowledgements}

The authors thank Dr Vyankatesh Shivane and Dr Neelam Jaguste for their assistance in conducting the research.

\section{References}

1 Gessler M, König A \& Bruns GA. The genomic organization and expression of the WT1 gene. Genomics 199212 807-813. (https://doi. org/10.1016/0888-7543(92)90313-h)

2 Reddy JC \& Licht JD. The WT1 Wilms' tumor suppressor gene: how much do we really know? Biochimica et Biophysica Acta $1996 \mathbf{1 2 8 7}$ 1-28. (https://doi.org/10.1016/0304-419x(95)00014-7)

3 Fischbach BV, Trout KL, Lewis J, Luis CA \& Sika M. WAGR syndrome: a clinical review of 54 cases. Pediatrics 2005116 984-988. (https://doi. org/10.1542/peds.2004-0467)

4 Pelletier J, Bruening W, Kashtan CE, Mauer SM, Manivel JC, Striegel JE, Houghton DC, Junien C, Habib R \& Fouser L. Germline mutations in the Wilms' tumor suppressor gene are associated with abnormal urogenital development in Denys-Drash syndrome. Cell 199167 437-447. (https://doi.org/10.1016/0092-8674(91)90194-4) 
5 Little M \& Wells C. A clinical overview of WT1 gene mutations. Human Mutation 19979 209-225. (https://doi.org/10.1002/(SICI)10981004(1997)9:3<209::AID-HUMU2>3.0.CO;2-2)

6 Barbaux S, Niaudet P, Gubler MC, Grünfeld JP, Jaubert F, Kuttenn F, Fékété CN, Souleyreau-Therville N, Thibaud E, Fellous M, et al. Donor splice-site mutations in WT1 are responsible for Frasier syndrome. Nature Genetics 199717 467-470. (https://doi.org/10.1038/ng1297-467)

7 Kohsaka T, Tagawa M, Takekoshi Y, Yanagisawa H, Tadokoro K \& Yamada M. Exon 9 mutations in the WT1 gene, without influencing KTS splice isoforms, are also responsible for Frasier syndrome. Human Mutation 199914 466-470. (https://doi.org/10.1002/(SICI)10981004(199912)14:6<466::AID-HUMU4>3.0.CO;2-6)

8 Köhler B, Biebermann H, Friedsam V, Gellermann J, Maier RF, Pohl M, Wieacker P, Hiort O, Grüters A \& Krude H. Analysis of the Wilms' tumor suppressor gene (WT1) in patients 46,XY disorders of sex development. Journal of Clinical Endocrinology and Metabolism 201196 E1131-E1136. (https://doi.org/10.1210/jc.2010-2804)

9 Sinnecker GH, Hiort O, Dibbelt L, Albers N, Dörr HG, Hauss H, Heinrich U, Hemminghaus M, Hoepffner W, Holder M, et al. Phenotypic classification of male pseudohermaphroditism due to steroid 5 alpha-reductase 2 deficiency. American Journal of Medical Genetics 199663 223-230. (https://doi.org/10.1002/(SICI)10968628(19960503)63:1<223::AID-AJMG39>3.0.CO;2-O)

10 Jeanpierre C, Denamur E, Henry I, Cabanis MO, Luce S, Cécille A, Elion J, Peuchmaur M, Loirat C, Niaudet P, et al. Identification of constitutional WT1 mutations, in patients with isolated diffuse mesangial sclerosis, and analysis of genotype/phenotype correlations by use of a computerized mutation database. American Journal of Human Genetics 199862 824-833. (https://doi org/10.1086/301806)

11 Fukuzawa R, Sakamoto J, Heathcott RW \& Hata JI. A necropsy case of Denys-Drash syndrome with a WT1 mutation in exon 7. Journal of Medical Genetics 200239 e48. (https://doi.org/10.1136/jmg.39.8.e48)

12 Auber F, Lortat-Jacob S, Sarnacki S, Jaubert F, Salomon R, Thibaud E, Jeanpierre C \& Nihoul-Fékété C. Surgical management and genotype/ phenotype correlations in WT1 gene-related diseases (Drash, Frasier syndromes). Journal of Pediatric Surgery 200338 124-129; discussion 124-129. (https://doi.org/10.1053/jpsu.2003.50025)

13 Maesaka A, Higuchi A, Kotoh S, Hasegawa Y, Ikeda M, Shishido S \& Honda M. Gonadal function in 15 patients associated with WT1 gene mutations. Clinical Pediatric Endocrinology 200615 143-149. (https:// doi.org/10.1297/cpe.15.143)

14 Regev M, Kirk R, Mashevich M, Bistritzer Z \& Reish O. Vertical transmission of a mutation in exon 1 of the WT1 gene: lessons for genetic counseling. American Journal of Medical Genetics: Part A 2008 146A 2332-2336. (https://doi.org/10.1002/ajmg.a.32330)

15 Nso Roca AP, Peña Carrión A, Benito Gutiérrez M, García Meseguer C, García Pose A \& Navarro M. Evolutive study of children with diffuse mesangial sclerosis. Pediatric Nephrology 200924 1013-1019. (https:// doi.org/10.1007/s00467-008-1063-z)

16 Tanteles GA, Oakley S, Christian M, O'neill D \& Suri M. DenysDrash syndrome and gonadoblastoma in a patient with Klinefelter syndrome. Clinical Dysmorphology 201120 131-135. (https://doi. org/10.1097/MCD.0b013e328346f6dc)

17 Patel PR, Pappas J, Arva NC, Franklin B \& Brar PC. Early presentation of bilateral gonadoblastomas in a Denys-Drash syndrome patient: a cautionary tale for prophylactic gonadectomy. Journal of Pediatric Endocrinology and Metabolism 201326 971-974. (https://doi. org/10.1515/jpem-2012-0409)

18 Mazen I, Hassan H, Kamel A, Mekkawy M, McElreavey K \& Essawi M. WT1 gene mutation, p.R462W, in a 46,XY DSD patient from Egypt with gonadoblastoma and review of the literature. Sexual Development 201711 280-283. (https://doi.org/10.1159/000485394)

19 Souza PVS, Badia BML, Silva LHL, Teixeira CAC, Seneor DD, Marin VDGB, Farias IB, Dias RB, Oliveira ASB \& Pinto WBVR. Leukodystrophy with disorders of sex development due to WT1 mutations. Journal of the Neurological Sciences 2018390 94-98. (https:// doi.org/10.1016/j.jns.2018.04.020)

20 Baxter RM, Arboleda VA, Lee H, Barseghyan H, Adam MP, Fechner PY, Bargman R, Keegan C, Travers S, Schelley S, et al. Exome sequencing for the diagnosis of 46,XY disorders of sex development. Journal of Clinical Endocrinology and Metabolism 2015100 E333-E344. (https:// doi.org/10.1210/jc.2014-2605)

21 Eggers S, Sadedin S, van den Bergen JA, Robevska G, Ohnesorg T, Hewitt J, Lambeth L, Bouty A, Knarston IM, Tan TY, et al. Disorders of sex development: insights from targeted gene sequencing of a large international patient cohort. Genome Biology 201617 243. (https:// doi.org/10.1186/s13059-016-1105-y)

22 Hughes LA, McKay-Bounford K, Webb EA, Dasani P, Clokie S, Chandran H, McCarthy L, Mohamed Z, Kirk JMW, Krone NP, et al. Next generation sequencing (NGS) to improve the diagnosis and management of patients with disorders of sex development (DSD). Endocrine Connections 20198 100-110. (https://doi.org/10.1530/EC-18-0376)

23 Wang H, Zhang L, Wang N, Zhu H, Han B, Sun F, Yao H, Zhang Q, Zhu W, Cheng T, et al. Next-generation sequencing reveals genetic landscape in 46,XY disorders of sexual development patients with variable phenotypes. Human Genetics 2018137 265-277. (https://doi. org/10.1007/s00439-018-1879-y)

24 Muto R, Yamamori S, Ohashi H \& Osawa M. Prediction by FISH analysis of the occurrence of Wilms tumor in aniridia patients. American Journal of Medical Genetics 2002108 285-289. (https://doi. org/10.1002/ajmg.10094)

25 Çatlı G, Alparslan C, Can PŞ, Akbay S, Kelekçi S, Atik T, Özyılmaz B $\&$ Dündar BN. An unusual presentation of $46, \mathrm{XY}$ pure gonadal dysgenesis: spontaneous breast development and menstruation. Journal of Clinical Research in Pediatric Endocrinology 20157 159-162. (https://doi.org/10.4274/jcrpe.1919)

26 Hall G, Gbadegesin RA, Lavin P, Wu G, Liu Y, Oh EC, Wang L, Spurney RF, Eckel J, Lindsey T, et al. A novel missense mutation of Wilms' tumor 1 causes autosomal dominant FSGS. Journal of the American Society of Nephrology 201526 831-843. (https://doi. org/10.1681/ASN.2013101053)

27 Ezaki J, Hashimoto K, Asano T, Kanda S, Akioka Y, Hattori M, Yamamoto T \& Shibata N. Gonadal tumor in Frasier syndrome: a review and classification. Cancer Prevention Research 20158 271-276. (https://doi.org/10.1158/1940-6207.CAPR-14-0415)

28 Hoei-Hansen CE. Application of stem cell markers in search for neoplastic germ cells in dysgenetic gonads, extragonadal tumours, and in semen of infertile men. Cancer Treatment Reviews 2008 34 348-367. (https://doi.org/10.1016/j.ctrv.2007.12.007)

29 Cools M, Looijenga LHJ, Wolffenbuttel KP \& T'Sjoen G. Managing the risk of germ cell tumourigenesis in disorders of sex development patients. Endocrine Development 201427 185-196. (https://doi. org/10.1159/000363642)

30 Schumacher V, Gueler B, Looijenga LHJ, Becker JU, Amann K, Engers R, Dotsch J, Stoop H, Schulz W \& Royer-Pokora B. Characteristics of testicular dysgenesis syndrome and decreased expression of SRY and SOX9 in Frasier syndrome. Molecular Reproduction and Development 200875 1484-1494. (https://doi.org/10.1002/mrd.20889)

31 Anna A \& Monika G. Splicing mutations in human genetic disorders: examples, detection, and confirmation. Journal of Applied Genetics 2018 59 253-268. (https://doi.org/10.1007/s13353-018-0444-7) https://ec.bioscientifica.com https://doi.org/10.1530/EC-21-0289 (c) 2021 The authors Published by Bioscientifica Ltd
Received in final form 21 September 2021

Accepted 2 November 2021

Accepted Manuscript published online 2 November 2021

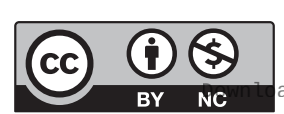

This work is licensed under a Creative Commons Attribution-NonCommercial 4.0 International License. ded from Bioscientifica com at $04 / 26 / 2023$ 12:12:56 AM 